\title{
Symmetric SOR Method for Absolute Complementarity Problems
}

\author{
Javed Iqbal and Muhammad Arif \\ Department of Mathematics, Abdul Wali Khan University Mardan, Mardan 23200, Pakistan \\ Correspondence should be addressed to Muhammad Arif; marifmaths@yahoo.com
}

Received 21 April 2013; Revised 3 August 2013; Accepted 19 August 2013

Academic Editor: Filomena Cianciaruso

Copyright (C) 2013 J. Iqbal and M. Arif. This is an open access article distributed under the Creative Commons Attribution License, which permits unrestricted use, distribution, and reproduction in any medium, provided the original work is properly cited.

\begin{abstract}
We study symmetric successive overrelaxation (SSOR) method for absolute complementarity problems. Solving this problem is equivalent to solving the absolute value equations. Some examples are given to show the implementation and efficiency of the method.
\end{abstract}

\section{Introduction}

Absolute complementarity problem seeks real vectors $x \geq 0$ and $A x-|x|-b \geq 0$, such that

$$
\langle x, A x-|x|-b\rangle=0,
$$

where $A \in R^{n \times n}$ and $b \in R^{n}$. The complementarity theory was introduced and studied by Lemke [1] and Cottle and Dantzig [2]. The complementarity problems have been generalized and extended to study a wide class of problems, which arise in pure and applied sciences; see [1-9] and the references therein. Equally important is the variational inequality problem, which was introduced and studied in the early sixties.

In this paper, we suggest and analyze SSOR [5] method for absolute complementarity problem which was introduced by Noor et al. [10]. The convergence analysis of the proposed method is considered under some suitable conditions. We show that the absolute complementarity problems are equivalent to variational inequalities. Results are very encouraging. The ideas and the technique of this paper may stimulate further research in these areas.

Let $R^{n}$ be the finite dimension Euclidean space, whose the inner product and norm are denoted by $\langle\cdot, \cdot\rangle\rangle$ and $\|\cdot\|$, respectively. For a given matrix $A \in R^{n \times n}$, a vector $b \in R^{n}$, we consider the problem of finding $x \in K^{*}$, such that

$$
\begin{gathered}
x \in K^{*}, \quad A x-|x|-b \in K^{*}, \\
\langle A x-|x|-b, x\rangle=0,
\end{gathered}
$$

where $K^{*}=\left\{x \in R^{n}:\langle x, y\rangle \geq 0, y \in K\right\}$ is the polar cone of a closed convex cone $K$ in $R^{n}$ and $|x|$ will denote the vector in $R^{n}$ with absolute values of components of $x \in R^{n}$. We remark that the absolute value complementarity problem (2) can be viewed as an extension of the complementarity problem considered by Karamardian [6].

Let $K$ be a closed and convex set in the inner product space $R^{n}$. We consider the problem of finding $x \in K$ such that

$$
\langle A x-|x|-b, y-x\rangle \geq 0, \quad \forall y \in K .
$$

The problem (3) is called the absolute value variational inequality, which is a special form of the mildly nonlinear variational inequalities [11]. If $K=R^{n}$, then the problem (3) is equivalent to find $x \in R^{n}$ such that

$$
A x-|x|-b=0 .
$$

To propose and analyze algorithms for absolute complementarity problems, we need the following definitions.

Definition 1. $B \in R^{n \times n}$ is called an $L$-matrix if $b_{i i}>0$ for $i=$ $1,2, \ldots, n$, and $b_{i j} \leq 0$ for $i \neq j, i, j=1,2, \ldots, n$. 
Definition 2. If $A \in R^{n \times n}$ is positive definite, then

(i) there exists a constant $\gamma>0$, such that

$$
\langle A x, x\rangle \geq \gamma\|x\|^{2}, \quad \forall x \in R^{n}
$$

(ii) there exists a constant $\beta>0$ such that

$$
\|A x\| \leq \beta\|x\|, \quad \forall x \in R^{n} .
$$

\section{Absolute Complementarity Problems}

To propose and analyze algorithm for absolute complementarity problems, we need the following results.

Lemma 3 (see [12]). Let $K$ be a nonempty closed convex set in $R^{n}$. For a given $z \in R^{n}, u \in K$ satisfies the inequality

$$
\langle u-z, u-v\rangle \geq 0, \quad v \in K,
$$

if and only if

$$
u=P_{K} z
$$

where $P_{K}$ is the projection of $R^{n}$ onto the closed convex set $K$.

Lemma 4 (see [10]). If $K$ is the positive cone in $R^{n}$, then $x \in K$ is a solution of absolute variational inequality (3) if and only if $x \in K$ is the solution of the complementarity problem (2).

The next result proves the equivalence between variational inequality (3) and the fixed point.

Lemma 5 (see [10]). If $K$ is closed convex set in $R^{n}$, then $\rho>0$; $x \in K$ satisfies (3) if and only if $x \in K$ satisfies the relation

$$
x=P_{K}(x-\rho[A x-|x|-b]),
$$

where $P_{K}$ is the projection of $R^{n}$ onto the closed convex set $K$.

Now using Lemmas 4 and 5, the absolute complementarity problem (2) can be transformed to fixed-point problem as

$$
x=P_{K}(x-\rho[A x-|x|-b]) .
$$

Theorem 6 (see [10]). Let $A \in R^{n \times n}$ be a positive definite matrix with constant $\alpha>0$ and continuous with constant $\beta>0$. If $0<\rho<2(\gamma-1) /\left(\beta^{2}-1\right), \beta>1, \gamma>1$, then there exists a unique solution $x \in K$, such that

$$
\langle A x-|x|-b, y-x\rangle \geq 0 \quad \forall y \in K,
$$

where $K$ is a closed convex set in $R^{n}$.

To define the projection operator $P_{K}$, we consider the special case when $K=[0, c]$ is a closed convex set in $R^{n}$, as follows.

Definition 7 (see [3]). Let $K=[0, c]$ is a closed convex set in $R^{n}$. Then, the projection operator $P_{K} x$ is defined as

$$
\left(P_{K} x\right)_{i}=\min \left\{\max \left(0, x_{i}\right), c_{i}\right\}, \quad i=1,2, \ldots, n .
$$

Lemma 8 (see [3]). For any $x$ and $y$ in $R^{n}$, the following facts hold:

(i) $P_{K}(x+y) \leq P_{K} x+P_{K} y$;

(ii) $P_{K} x-P_{K} y \leq P_{K}(x-y)$;

(iii) $x \leq y \Rightarrow P_{K} x \leq P_{K} y$;

(iv) $P_{K} x+P_{K}(-x) \leq|x|$, with equality, if and only if $-c \leq x \leq c$

Now one splits the matrix $A$ as

$$
A=D-L-U
$$

where $D$ is the diagonal matrix and $L$ and $U$ are strictly lower and strictly upper triangular matrices, respectively. Let $0<\omega<$ 2; using (13), one suggests the SSOR method for solving (3) as follows.

Algorithm 9. Consider the following.

Step 1. Choose an initial vector $x_{0} \in R^{n}$ and a parameter $\omega \epsilon$ $R_{+}$. Set $k=0$.

Step 2. Calculate

$$
\begin{gathered}
x_{k+1}=P_{K}\left(x_{k}-D^{-1}\right. \\
\times\left[-\omega L x_{k+1}+(\omega(2-\omega) A+\omega L) x_{k}\right. \\
\left.\left.\quad-\omega(2-\omega)\left(\left|x_{k}\right|+b\right)\right]\right) .
\end{gathered}
$$

Step 3. If $x_{k+1}=x_{k}$, then stop; else, set $k=k+1$ and go to step 2.

Algorithm 10. Consider the following.

Step 1. Choose an initial vector $x_{0} \in R^{n}$ and a parameter $\omega \epsilon$ $R_{+}$. Set $k=0$.

Step 2. Calculate

$$
\begin{gathered}
x_{k+1}=P_{K}\left(x_{k}-D^{-1}\right. \\
\times\left[-\omega U x_{k+1}+(\omega(2-\omega) A+\omega U) x_{k}\right. \\
\left.\left.\quad-\omega(2-\omega)\left(\left|x_{k}\right|+b\right)\right]\right) .
\end{gathered}
$$

Step 3. If $x_{k+1}=x_{k}$, then stop; else, set $k=k+1$ and go to step 2.

Now we define an operator $g: R^{n} \rightarrow R^{n}$ such that $g(x)=$ $\xi$, where $\xi$ is the fixed point of the system

$$
\begin{gathered}
\xi=P_{K}\left(x-D^{-1}\right. \\
\times[-\omega L \xi+(\omega(2-\omega) A+\omega L) x \\
\quad-\omega(2-\omega)(|x|+b)]) .
\end{gathered}
$$

We also assume that the set

$$
\varphi=\left\{x \in R^{n}: x \geq 0, A x-|x|-b \geq 0\right\}
$$


of the absolute complementarity problem is nonempty. To prove the convergence of Algorithm 9, we need the following result.

Theorem 11. Consider the operator $g: R^{n} \rightarrow R^{n}$ as defined in (16). Assume that $A \in R^{n \times n}$ is an L-matrix. Also assume that $0<\omega \leq 1$. Then for any $x \in \varphi$, it holds that.

(i) $g(x) \leq x$;

(ii) $x \leq y \Rightarrow g(x) \leq g(y)$;

(iii) $\xi=g(x) \in \varphi$.

Proof. To prove (i), we need to prove that

$$
\xi_{i} \leq x_{i}, \quad i=1,2, \ldots, n
$$

with $\xi_{i}$ satisfying

$$
\begin{aligned}
\xi_{i}=P_{K}\left(x_{i}-a_{i i}^{-1}\right. & \\
\times & {\left[-\omega \sum_{j=1}^{i-1} L_{i j}\left(\xi_{j}-x_{j}\right)+\omega(2-\omega)\right.} \\
& \left.\left.\times(A x-|x|-b)_{i}\right]\right) .
\end{aligned}
$$

To prove the required result, we use mathematical induction. For this, let $i=1$ :

$$
\xi_{1}=P_{K}\left(x_{1}-a_{11}^{-1} \omega(2-\omega)(A x-|x|-b)_{1}\right) .
$$

Since $A x-|x|-b \geq 0,0<\omega \leq 1$; therefore, $\xi_{1} \leq x_{1}$. For $i=2$, we have

$$
\begin{aligned}
\xi_{2}=P_{K}\left(x_{2}-\right. & a_{22}^{-1} \\
\times & -\omega L_{21}\left(\xi_{1}-x_{1}\right)+\omega(2-\omega) \\
& \left.\left.\times(A x-|x|-b)_{2}\right]\right) .
\end{aligned}
$$

Here, $A x-|x|-b \geq 0,0<\omega \leq 2, L_{21} \geq 0$ and $\xi_{1}-x_{1} \leq 0$. This implies that $\xi_{2} \leq x_{2}$.

Suppose that

$$
\xi_{i} \leq x_{i} \text { for } i=1,2, \ldots, k-1,
$$

we have to prove that the statement is true for $i=k$; that is,

$$
\xi_{k} \leq x_{k}
$$

Consider

$$
\begin{array}{r}
\xi_{k}=P_{K}\left(x_{k}-a_{k k}^{-1}\right. \\
\times\left[-\omega \sum_{j=1}^{k-1} L_{k j}\left(\xi_{j}-x_{j}\right)+\omega(2-\omega)\right. \\
\left.\left.\times(A x-|x|-b)_{k}\right]\right) \\
=P_{K}\left(x_{k}-a_{k k}^{-1}\right. \\
\times\left[-\omega\left(L_{k 1}\left(\xi_{1}-x_{1}\right)+L_{k 2}\left(\xi_{2}-x_{2}\right)\right.\right. \\
\left.+\cdots+L_{k k-1}\left(\xi_{k-1}-x_{k-1}\right)\right) \\
\left.\left.+\omega(2-\omega)(A x-|x|-b)_{k}\right]\right) .
\end{array}
$$

Since $A x-|x|-b \geq 0,0<\omega_{k} \leq 2, L_{k 1}, L_{k 2}, \ldots, L_{k k-1} \geq 0$ and $\xi_{i} \leq x_{i}$ for $i=1,2, \ldots, k-1$; from (24), we can write

$$
\xi_{k} \leq x_{k} \text {. }
$$

Hence, (i) is proved.

Now we prove (ii), for this let us suppose that $\xi=g(x)$ and $\phi=g(y)$. We will prove that

$$
x \leq y \Longrightarrow \xi \leq \phi .
$$

As

$$
\begin{aligned}
\xi=P_{K}\left(x-D^{-1}\right. & \\
\times[-\omega L \xi+(\omega(2-\omega) A+\omega L) x & -\omega(2-\omega)(|x|+b)]),
\end{aligned}
$$

so $\xi_{i}$ can be written as

$$
\begin{aligned}
& \xi_{i}=P_{K}\left(-a_{i i}^{-1}\right. \\
& \times\left[-\omega \sum_{j=1}^{i-1} L_{i j} \xi_{j}+\omega a_{i i} x_{i}+(\omega-\omega(2-\omega))\right. \\
& \times \sum_{j=1}^{i-1} L_{i j} x_{j}-\omega(2-\omega) \\
& \times \sum_{j=1}^{n} U_{j \neq i} x_{j}-\omega(2-\omega)\left|x_{i}\right|
\end{aligned}
$$$$
\left.\left.-\omega(2-\omega) b_{i}\right]\right)
$$ 


$$
\begin{aligned}
& =P_{K}\left((1-\omega) x_{i}-a_{i i}^{-1}\right. \\
& \times\left[-\omega \sum_{j=1}^{i-1} L_{i j} \xi_{j}+(\omega-\omega(2-\omega))\right. \\
& \times \sum_{j=1}^{i-1} L_{i j} x_{j}-\omega(2-\omega) \\
& \times \sum_{j=1}^{n} U_{i j} x_{j}-\omega(2-\omega)\left|x_{i}\right| \\
& \left.\left.-\omega(2-\omega) b_{i}\right]\right) \text {. }
\end{aligned}
$$

Similarly, for $\phi_{i}$ we have

$$
\begin{gathered}
\phi_{i}=P_{K}\left((1-\omega) y_{i}-a_{i i}^{-1}\right. \\
\times\left[-\omega \sum_{j=1}^{i-1} L_{i j} \phi_{j}+(\omega-\omega(2-\omega))\right. \\
\times \sum_{j=1}^{i-1} L_{i j} y_{j}-\omega(2-\omega) \\
\times \sum_{j=1 j \neq i}^{n} U_{i j} y_{j}-\omega(2-\omega)\left|y_{i}\right| \\
\left.\left.-\omega(2-\omega) b_{i}\right]\right) .
\end{gathered}
$$

For $i=1$, we have

$$
\begin{aligned}
\phi_{1}=P_{K}( & (1-\omega) y_{1}-a_{11}^{-1} \omega(2-\omega) \\
& \left.\times\left[-\sum_{j=1}^{n} U_{1 j} y_{j}-\left|y_{1}\right|-b_{1}\right]\right) \\
\geq P_{K}( & (1-\omega) x_{1}-a_{11}^{-1} \omega(2-\omega) \\
& \left.\times\left[-\sum_{j=1}^{n} U_{1 j} x_{j}-\left|x_{1}\right|-b_{1}\right]\right) \\
& =\xi_{1} .
\end{aligned}
$$

Since $y_{1} \geq x_{1}$, therefore $-\left|y_{1}\right| \leq-\left|x_{1}\right|$. Hence, it is true for $i=1$. Suppose it is true for $i=1,2, \ldots, k-1$; we will prove it for $i=k$; for this consider

$$
\begin{aligned}
& \phi_{k}=P_{K}\left((1-\omega) y_{k}-a_{k k}^{-1}\right. \\
& \times {\left[\sum_{j=1}^{k-1} L_{k j} \phi_{j}+(\omega-\omega(2-\omega))\right.} \\
& \times \sum_{j=1}^{k-1} L_{k j} y_{j}-\omega(2-\omega) \\
& \times \sum_{j=1 j \neq i}^{n} U_{k j} y_{j}-\omega(2-\omega)\left|y_{k}\right|
\end{aligned}
$$

$$
\begin{aligned}
& \geq P_{K}\left((1-\omega) x_{k}-a_{k k}^{-1}\right. \\
& \quad \times\left[\sum_{j=1}^{k-1} L_{k j} \xi_{j}+(\omega-\omega(2-\omega))\right. \\
& \quad \times \sum_{j=1}^{k-1} L_{k j} x_{j}-\omega(2-\omega) \\
& \times \sum_{j=1 j \neq i}^{n} U_{k j} x_{j}-\omega(2-\omega)\left|x_{k}\right| \\
& \left.\left.\quad-\omega(2-\omega) b_{k}\right]\right)
\end{aligned}
$$$$
=\xi_{k} \text {. }
$$

Since $x \leq y$ and $\xi_{i} \leq \phi_{i}$ for $i=1,2, \ldots, k-1$, hence it is true for $k$ and (ii) is verified.

Next we prove (iii); that is,

$$
\xi=g(x) \in \varphi .
$$

Let $\lambda=g(\xi)=P_{K}\left(\xi-D^{-1}[\omega L(\lambda-\xi)+\omega(2-\omega)(A \xi-|\xi|-\right.$ b)]) from (i) $g(\xi)=\lambda \leq \xi$. Also by definition of $g, \xi=g(x) \geq$ 0 and $\lambda=g(\xi) \geq 0$.

Now

$$
\begin{aligned}
\lambda_{i}=P_{K}\left(\xi_{i}-a_{i i}^{-1}\right. & \\
\times & {\left[-\omega \sum_{j=1}^{i-1} L_{i j}\left(\lambda_{j}-\xi_{j}\right)+\omega(2-\omega)\right.} \\
& \left.\left.\times(A \xi-|\xi|-b)_{i}\right]\right)
\end{aligned}
$$


For $i=1, \xi_{1} \geq 0$ by definition of $g$. Suppose that $(A \xi-|\xi|-$ $b)_{i}<0$, so

$$
\begin{aligned}
\lambda_{1} & =P_{K}\left(\xi_{1}-a_{11}^{-1} \omega(2-\omega)(A \xi-|\xi|-b)_{1}\right) \\
& >P_{K}\left(\xi_{1}\right)=\xi_{1},
\end{aligned}
$$

which contradicts the fact that $\lambda \leq \xi$. Therefore, $(A \xi-|\xi|-$ $b)_{i} \geq 0$.

Now we prove it for any $k$ in $i=1,2, \ldots, n$. Suppose the contrary $(A \xi-|\xi|-b)_{i}<0$; then

$$
\begin{aligned}
& \lambda_{k}=P_{K}\left(\xi_{k}-a_{k k}^{-1}\right. \\
& \times {\left[-\omega \sum_{j=1}^{k-1} L_{k j}\left(\lambda_{j}-\xi_{j}\right)+\omega(2-\omega)\right.} \\
&\left.\left.\times(A \xi-|\xi|-b)_{k}\right]\right) .
\end{aligned}
$$

As it is true for all $\alpha \in[0,1]$, it should be true for $\alpha=0$. That is,

$$
\begin{aligned}
\lambda_{k} & =P_{K}\left(\xi_{k}-a_{k k}^{-1} \omega(2-\omega)(A \xi-|\xi|-b)_{k}\right) \\
& >P_{K}\left(\xi_{k}\right)=\xi_{k},
\end{aligned}
$$

which contradicts the fact that $\lambda \leq \xi$. So, $(A \xi-|\xi|-b)_{k} \geq 0$, for any $k$ in $i=1,2, \ldots, n$.

Hence, $\xi=f(x) \in \varphi$.

Now we prove the convergence criteria of Algorithm 9 when the matrix $A$ is an $L$-matrix as stated in the next result.

Theorem 12. Assume that $A \in R^{n \times n}$ is an L-matrix and $0<$ $\omega \leq 1$. Then for any initial vector $x_{0} \in \varphi$, the sequence $\left\{x_{k}\right\}, k=$ $0,1,2, \ldots$, defined by Algorithm 9 has the following properties:

(i) $0 \leq x_{k+1} \leq x_{k} \leq x_{0} ; k=0,1,2, \ldots$;

(ii) $\lim _{k \rightarrow \infty} x_{k}=x^{*}$ is the unique solution of the absolute complementarity problem.

Proof. Since $x_{0} \in \varphi$, by (i) of Theorem 11, we have $x_{1} \leq x_{0}$ and $x_{1} \in \varphi$. Recursively using Theorem 11, we obtain

$$
0 \leq x_{k+1} \leq x_{k} \leq x_{0} ; \quad k=0,1,2, \ldots
$$

From (i), we observe that the sequence $\left\{x_{k}\right\}$ is monotone bounded; therefore, it converges to some $x^{*} \in R^{n}$, satisfying

$$
\begin{gathered}
x^{*}=P_{K}\left(x^{*}-D^{-1}\right. \\
\times\left[-\omega L x^{*}+\omega(2-\omega)(A+\omega L) x^{*}\right. \\
\left.\left.-\omega(2-\omega)\left(\left|x^{*}\right|+b\right)\right]\right) \\
=P_{K}\left(x^{*}-D^{-1} \omega(2-\omega)\right. \\
\left.\times\left[A x^{*}-\left|x^{*}\right|-b\right]\right) .
\end{gathered}
$$

Hence, $x^{*}$ is the solution of the absolute complementarity problem (2).

Note. The convergence of Algorithm 10 has the same steps as given in Theorems 11 and 12 .

\section{Numerical Results}

In this section, we consider several examples to show the efficiency of the proposed methods. The convergence of SSOR method is guaranteed for $L$-matrices only, but it is also possible to solve different type of systems. All the experiments are performed with Intel(R) Core $2 \times 2.1 \mathrm{GHz}, 1 \mathrm{~GB}$ RAM, and the codes are written in MATLAB 7.

Example 13 (see [10]). Consider the ordinary differential equation

$$
\begin{gathered}
\frac{d^{2} x}{d t^{2}}-|x|=\left(1-t^{2}\right), \quad 0 \leq x \leq 1, \\
x(0)=0 \quad x(1)=1
\end{gathered}
$$

The exact solution is

$x(t)= \begin{cases}0.7378827425 \sin (t)-3 \cos (t)+3-t^{2} & x<0, \\ -0.7310585786 e^{-t}-0.2689414214 e^{t}+1+t^{2} & x>0 .\end{cases}$

We take $n=10$; the matrix $A$ is given by

$$
a_{i, j}= \begin{cases}-242, & \text { for } j=i \\ 121 & \text { for } \begin{cases}j=i+1, & i=1,2, \ldots, n-1 \\ j=i-1, & i=2,3, \ldots, n\end{cases} \\ 0, & \text { otherwise. }\end{cases}
$$

The constant vector $b$ is given by

$$
b=\left(\frac{120}{121}, \frac{117}{121}, \frac{112}{121}, \frac{105}{121}, \frac{96}{121}, \frac{85}{121}, \frac{72}{121}, \frac{57}{121}, \frac{40}{121}, \frac{-14620}{121}\right)^{T} .
$$

Here, $A$ is not an $L$-matrix. The comparison between the exact solution and the approximate solutions is given in Figure 1.

In Figure 1, we see that the SSOR method converges rapidly to the approximate solution of absolute complementarity problem (2) as compared to GAOR method.

In the next example, we compare SSOR method with iterative method by Noor et al. [13].

Example 14 (see [13]). Let the matrix $A$ be given by

$$
a_{i, j}= \begin{cases}8, & \text { for } j=i \\ -1 & \text { for } \begin{cases}j=i+1, & i=1,2, \ldots, n-1 \\ j=i-1, & i=2,3, \ldots, n\end{cases} \\ 0, & \text { otherwise. }\end{cases}
$$

Let $b=(6,5,5, \ldots, 5,6)^{T}$, the problem size $n$, ranging from 4 to 1024 . The stopping criteria are $\|A x-|x|-b\|<10^{-6}$. 


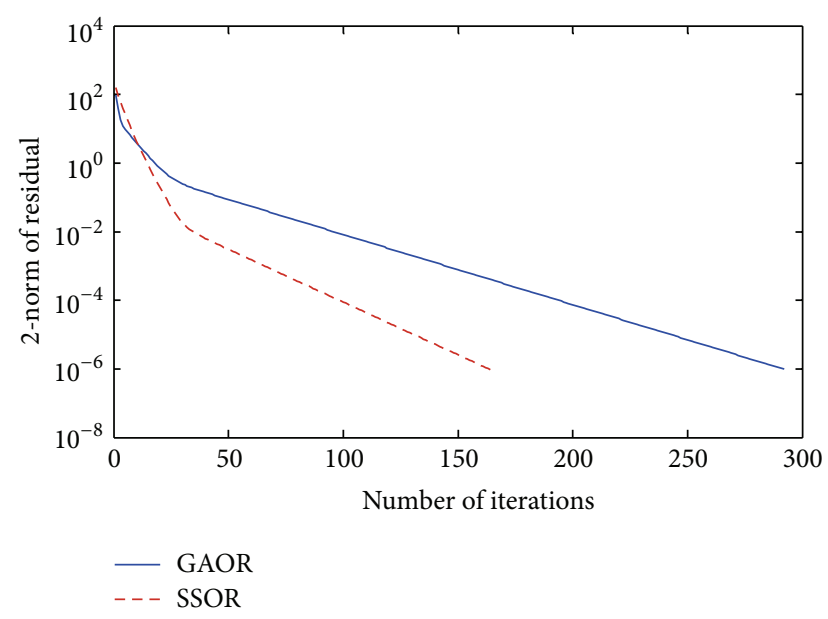

FIGURE 1: Comparison between GAOR method and SSOR method.

TABLE 1

\begin{tabular}{lcccc}
\hline$n$ & \multicolumn{2}{c}{ Iterative method } & \multicolumn{2}{c}{ SSOR method } \\
& Number of iterations & TOC & Number of iterations & TOC \\
\hline 4 & 10 & 0.0168 & 8 & 0.001 \\
8 & 11 & 0.018 & 8 & 0.001 \\
16 & 11 & 0.143 & 9 & 0.001 \\
32 & 12 & 3.319 & 9 & 0.001 \\
64 & 12 & 7.145 & 9 & 0.015 \\
128 & 12 & 11.342 & 9 & 0.020 \\
256 & 12 & 25.014 & 10 & 1.051 \\
512 & 12 & 98.317 & 10 & 6.130 \\
1024 & 13 & 534.903 & 10 & 126.242 \\
\hline
\end{tabular}

We choose initial guess $x_{0}$ as $x_{0}=(0,0, \ldots, 0)^{T}$. The computational results are shown in Table 1.

In Table 1, TOC denotes the total time taken by CPU. The rate of convergence of SSOR method is better than that of iterative method [13].

\section{Conclusion}

In this paper, we have discussed symmetric SOR method for solving absolute complementarity problem. The comparison with other methods showed the efficiency of the method. The results and ideas of this paper may be used to solve the variational inequalities and related optimization problems.

\section{References}

[1] C. E. Lemke, "Bimatrix equilibrium points and mathematical programming," Management Science, vol. 11, pp. 681-689, 1965.

[2] R. W. Cottle and G. B. Dantzig, "Complementary pivot theory of mathematical programming," Linear Algebra and its Applications, vol. 1, no. 1, pp. 103-125, 1968.

[3] B. H. Ahn, "Iterative methods for linear complementarity problems with upperbounds on primary variables," Mathematical Programming, vol. 26, no. 3, pp. 295-315, 1983.
[4] R. W. Cottle, J.-S. Pang, and R. E. Stone, The Linear Complementarity Problem, Computer Science and Scientific Computing, Academic Press, Boston, Mass, USA, 1992.

[5] M. Dehghan and M. Hajarian, "Convergence of SSOR methods for linear complementarity problems," Operations Research Letters, vol. 37, no. 3, pp. 219-223, 2009.

[6] S. Karamardian, "Generalized complementarity problem," Journal of Optimization Theory and Applications, vol. 8, pp. 161-168, 1971.

[7] Y. Li and P. Dai, "Generalized AOR methods for linear complementarity problem," Applied Mathematics and Computation, vol. 188, no. 1, pp. 7-18, 2007.

[8] O. L. Mangasarian, "Absolute value equation solution via concave minimization," Optimization Letters, vol. 1, no. 1, pp. 3-8, 2007.

[9] O. L. Mangasarian, "Solution of symmetric linear complementarity problems by iterative methods," Journal of Optimization Theory and Applications, vol. 22, no. 4, pp. 465-485, 1977.

[10] M. A. Noor, J. Iqbal, K. I. Noor, and E. Al-Said, "Generalized AOR method for solving absolute complementarity problems," Journal of Applied Mathematics, vol. 2012, Article ID 743861, 14 pages, 2012.

[11] M. A. Noor, On variational inequalities [Ph.D. thesis], Brunel University, London, UK, 1975.

[12] M. A. Noor, "On merit functions for quasivariational inequalities," Journal of Mathematical Inequalities, vol. 1, no. 2, pp. 259268, 2007.

[13] M. A. Noor, J. Iqbal, K. I. Noor, and E. Al-Said, "On an iterative method for solving absolute value equations," Optimization Letters, vol. 6, no. 5, pp. 1027-1033, 2012. 


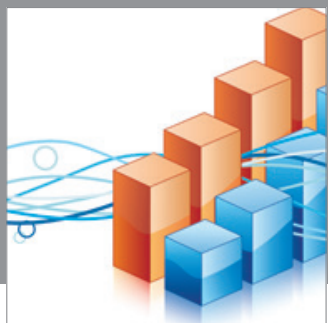

Advances in

Operations Research

mansans

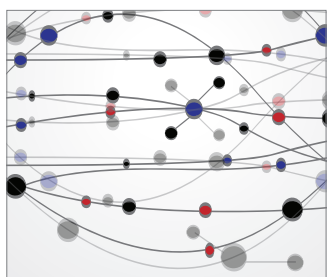

The Scientific World Journal
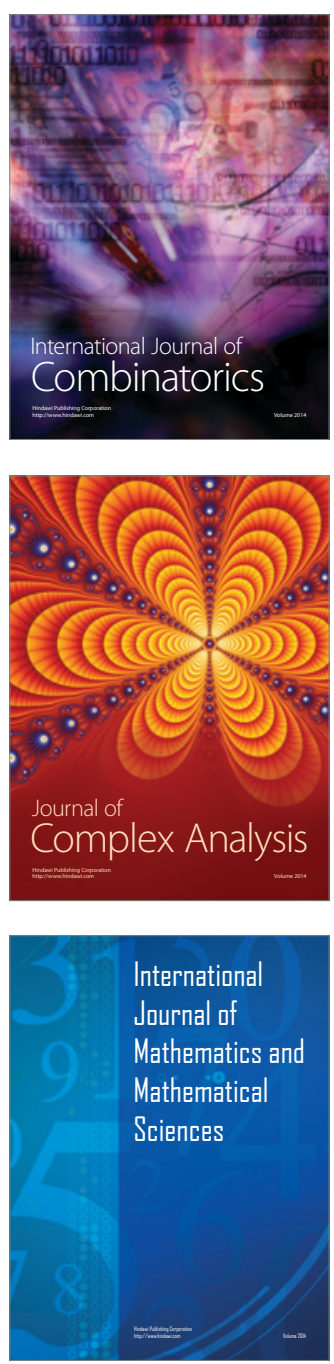
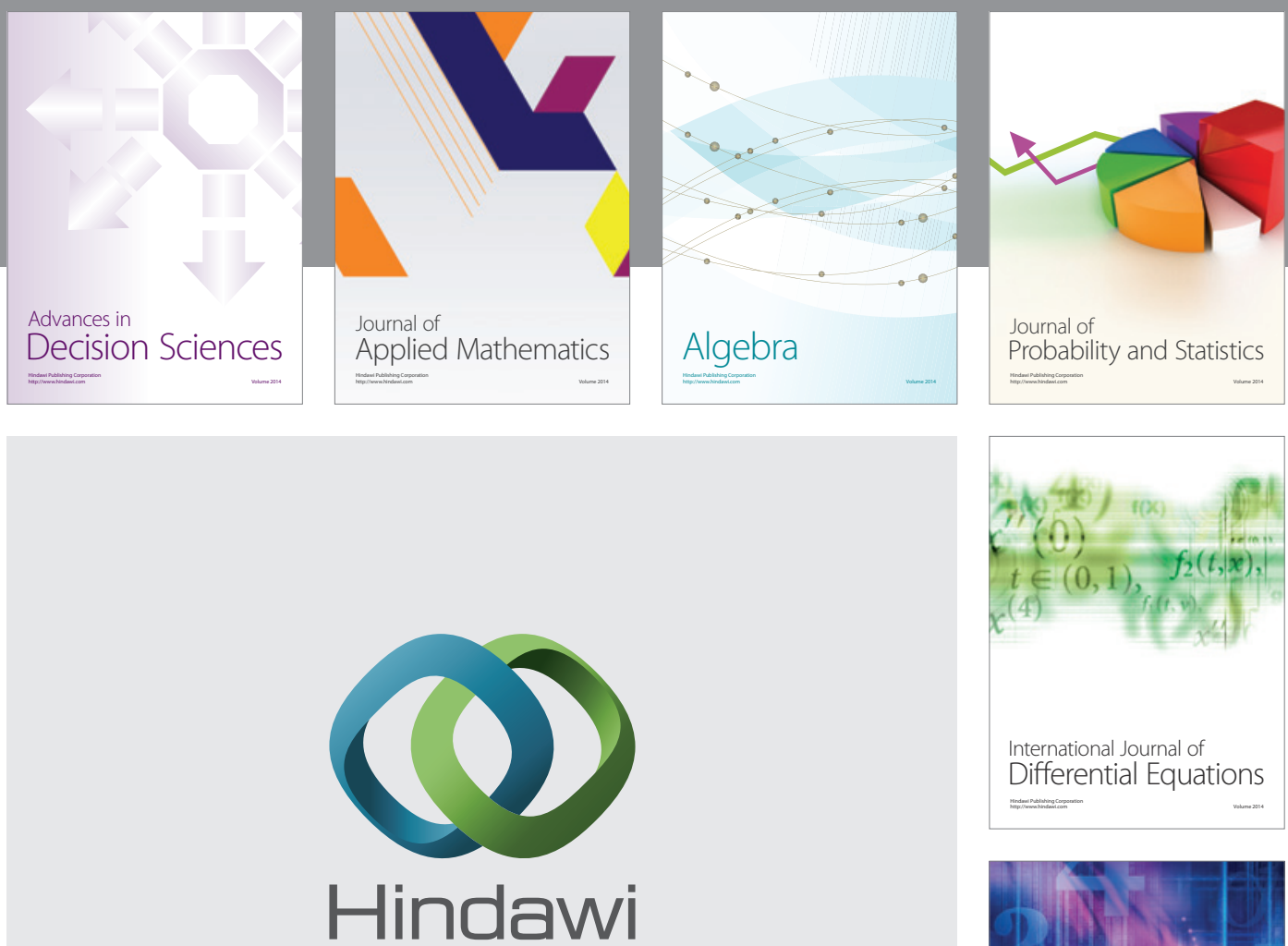

Submit your manuscripts at http://www.hindawi.com
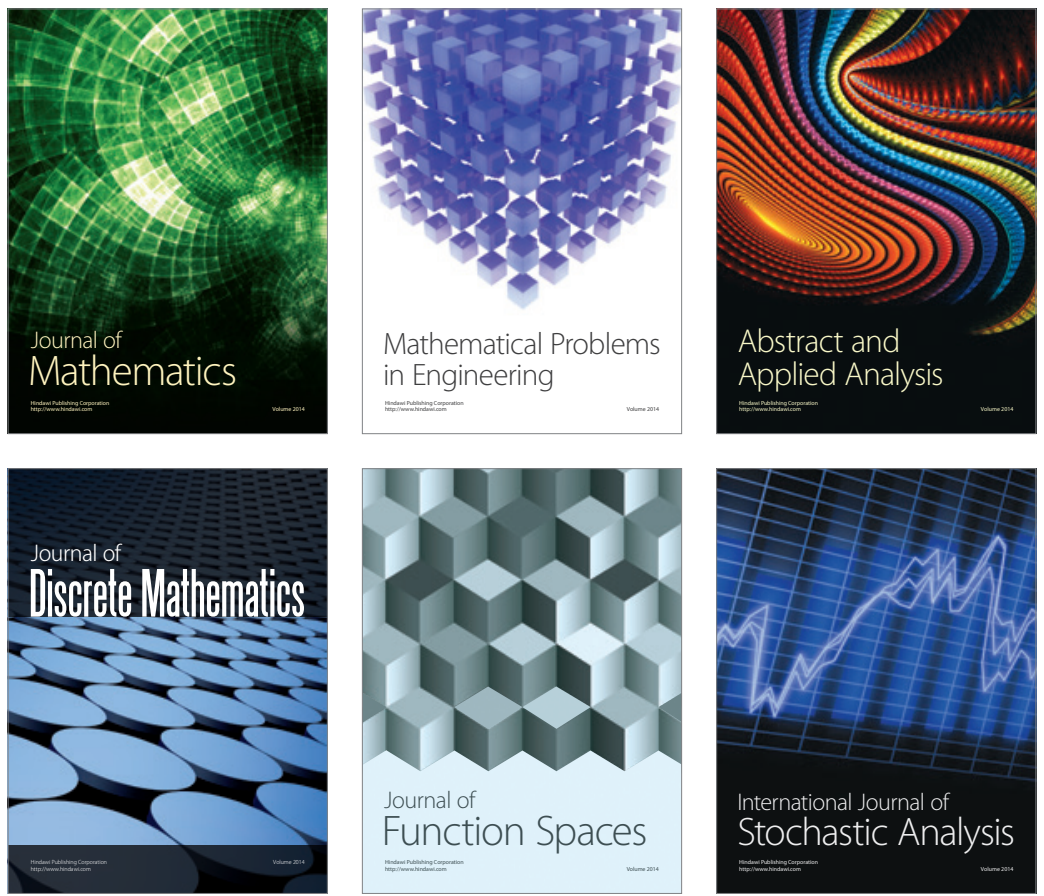

Journal of

Function Spaces

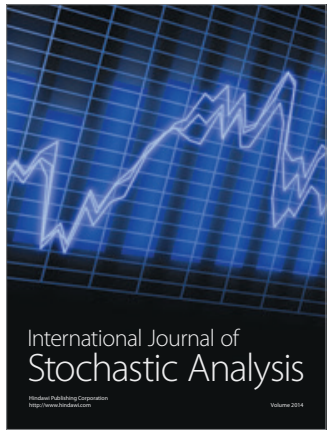

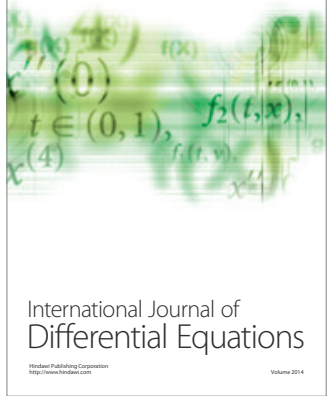
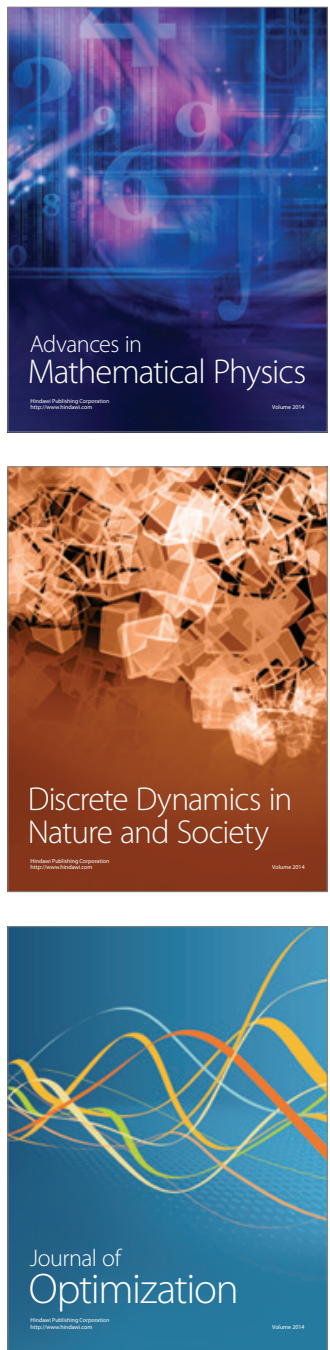